\title{
Effects of repeated administration of amitriptyline on modulation of noradrenergic descending inhibitory system in a rat model of neuropathic pain
}
Hiroki Tadanao. ${ }^{1}$, Suto Takashi. ${ }^{1}$, Saito Shigeru. ${ }^{1}$, Obata Hideaki. ${ }^{2}$
1) Gunma University, Dept of Anesthesiology, Maebashi, Japan
2) Fukushima Medical University Hospital, Center of Pain Management, Fukushima, Japan

\section{Background and Goal of Study \\ The tricyclic antidepressant amitriptyline, the serotonin and noradrenaline reuptake inhibitor duloxetine, and gabapentinoids are first-line drug for treatment of neuropathic pain. The analgesic effect of these drugs relates to brainstem-spinal descending noradrenergic (NA) systems. In the present study, we investigated the difference in NA dependency of each drug. We also examined whether amitriptyline modifies the descending NA systems.}

\section{Materials and methods}

Seven days after L5 spinal nerve ligation (SNL), rats received $\mathrm{N}$-(2-chloroethyl)-N-ethyl-2-bromobenzylamine (DSP-4, $50 \mathrm{mg} / \mathrm{kg}$ ) to degenerate NA fibers. The rats then received five daily intraperitoneal injections of amitriptyline $(10 \mathrm{mg} / \mathrm{kg})$, duloxetine $(10 \mathrm{mg} / \mathrm{kg})$, pregabalin $(10 \mathrm{mg} / \mathrm{kg})$, or gabapentin $(50 \mathrm{mg} / \mathrm{kg})$ from 21 days after SNL surgery. Paw withdrawal thresholds were determined to assess the analgesic effect of the drugs by paw pressure test. To determine whether five daily injections of amitriptyline activated NA neurons in the locus coeruleus (LC) and spinal cord, we performed immunohistochemistry using antibodies for c-Fos and dopamine beta-hydroxylase $(\mathrm{D} \beta \mathrm{H})$.

\begin{tabular}{|lll}
\hline $\begin{array}{l}\text { A Paw pressure test } \\
\text { training pre-SNL }\end{array}$ & A: Fig. 1 \\
SNL surgery DSP-4 or saline i.p. & \\
B Paw pressure test & Brug or vehicle i.p. 5 days & \\
training pre-SNL &
\end{tabular}

\section{Results and Discussion}

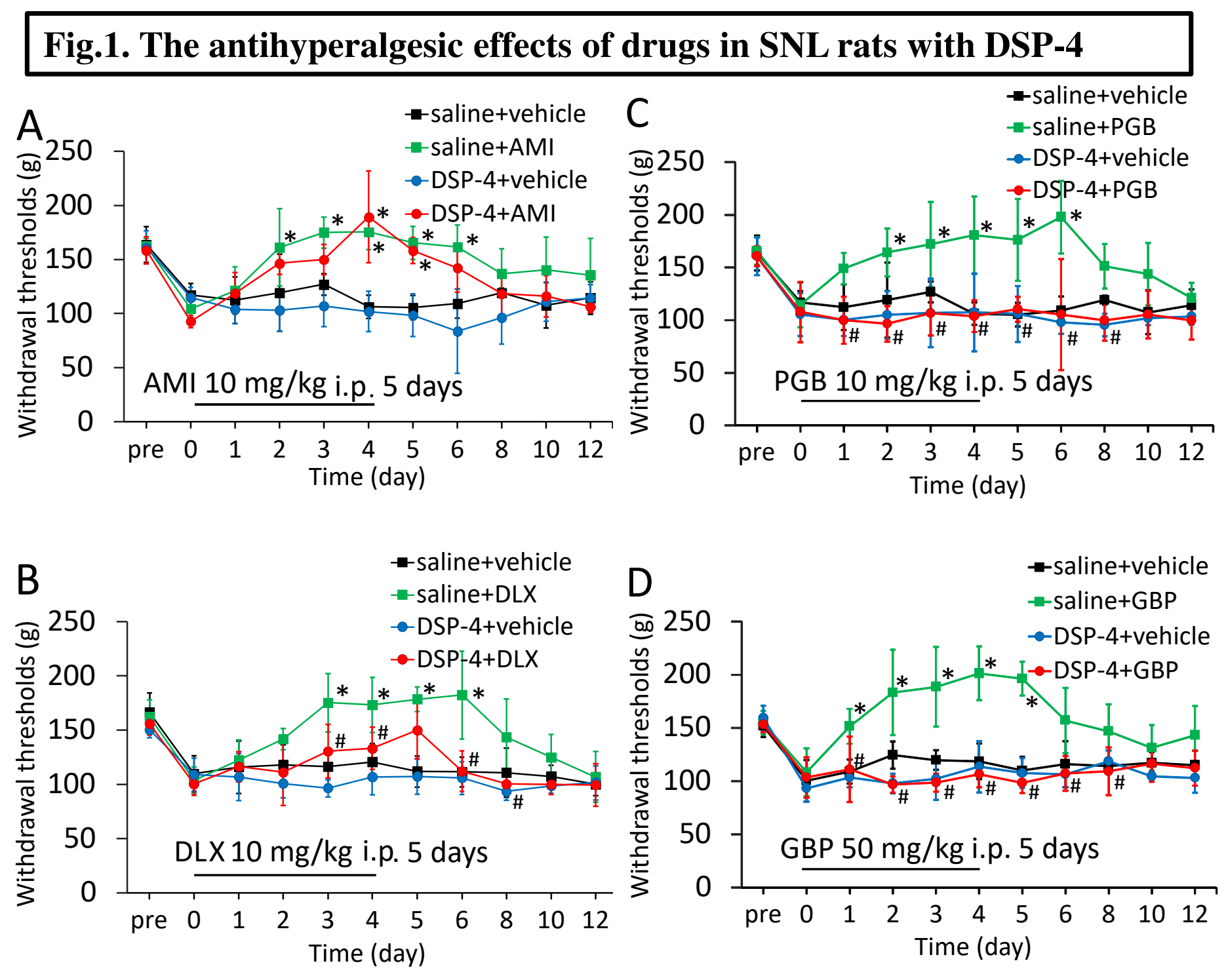

AMI: amitriptyline, DLX: duloxetine, PGB: pregabalin, GBP: gabapentin.

$\mathrm{N}=6$

$* \mathrm{P}<0.01$ vs the control (saline + vehicle) group

$\# \mathrm{P}<0.01$ vs the saline + drug group

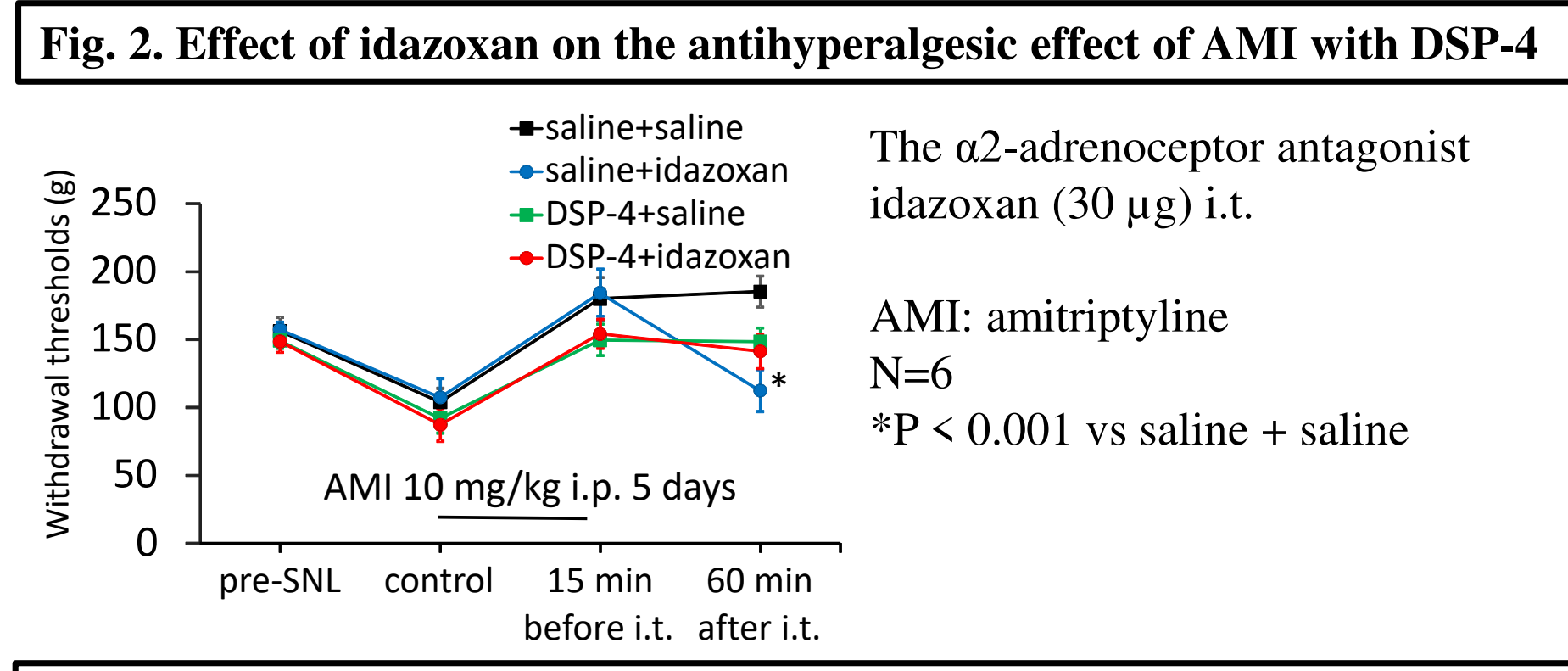

Fig. 3. Effects of AMI/DSP-4 on neuronal activity and DBH-IR fibers in LC

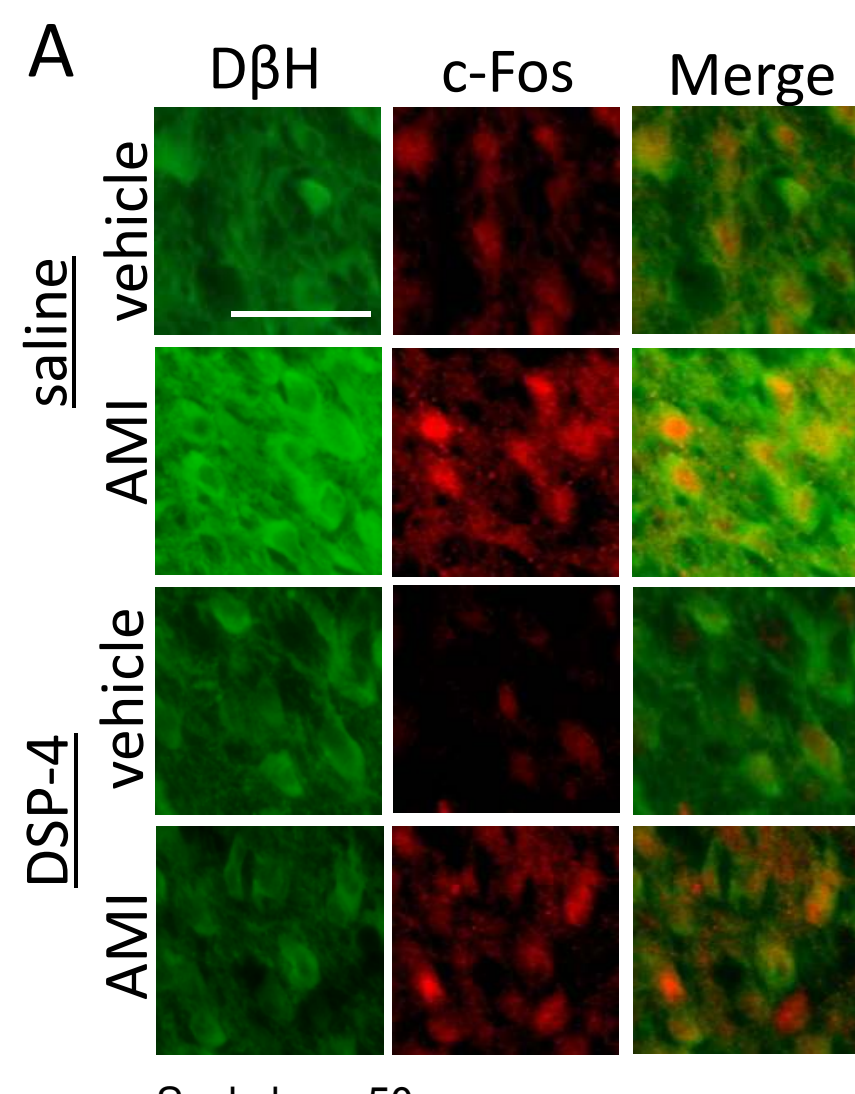

Scale bar $=50 \mu \mathrm{m}$

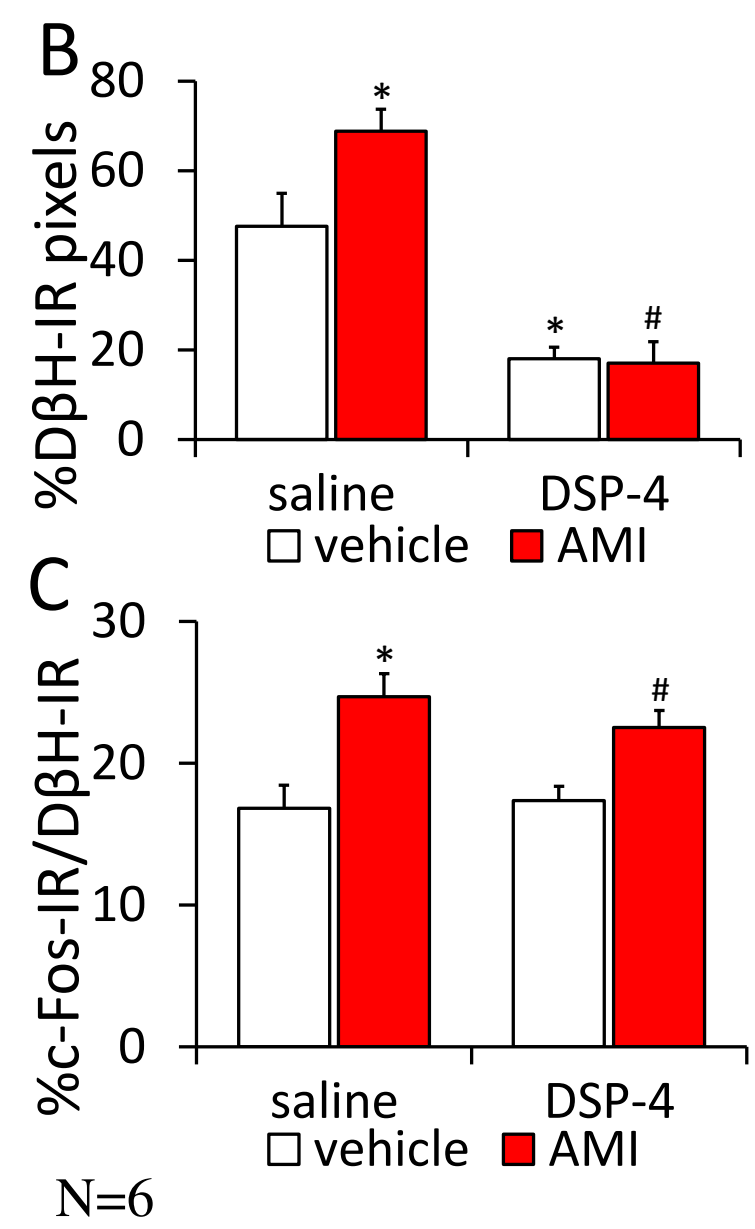

$* \mathrm{P}<0.001$ vs saline + vehicle \#P $<0.001$ vs DSP-4 and vehicle 